\title{
Megacraniectomy for Malignant Intracranial Hypertension: No Time for Caution
}

\author{
Prashant K. Chaudhary ${ }^{1}$ \\ ${ }^{1}$ Department of Neurosurgery, Sri Balaji Action Medical Institute \\ and Action Cancer Hospital, New Delhi, India \\ 2Department of Neurosurgery, Postgraduate Institute of Medical \\ Education and Research, Chandigarh, Punjab, India \\ ${ }^{3}$ Department of Neurosurgery, Sanjay Gandhi Postgraduate \\ Institute of Medical Sciences, Lucknow, Uttar Pradesh, India \\ J Neurosci Rural Pract 2019;10:555-558
}

\begin{abstract}
Address for correspondence Manjul Tripathi, MS, MCh, Department of Neurosurgery, Neurosurgery Office, Post Graduate Institute of Medical Education and Research, Chandigarh 160012, Punjab, India (e-mail: drmanjultripathi@gmail.com).
\end{abstract}

\begin{abstract}
Keywords

- intracranial hypertension

- megacraniectomy

- refractory

Malignant intracranial hypertension (IHT) intracranial tension (ICT) is a surgical emergency. Routine decompressive craniectomy may not be sufficient in reducing the malignant IHT. At present, we do not have the exact solution to this ominous situation. Authors came across a similar scenario where we had to go forward with modification of a previously known described procedure, removing bifrontal, temporal, and parietal bones including midline bone strip over a superior sagittal sinus in a case of resistant malignant ICT, following coiling of an anterior communicating artery aneurysm. This radical technique, named as megacraniectomy, was used as a last resort in a rapidly deteriorating patient. The patient survived the stormy phase of malignant ICT and showed significant improvement in neurological status. Authors here describe this approach as a novel idea to be explored in resource-stricken situations.
\end{abstract}

\section{Introduction}

Malignant intracranial hypertension (IHT) in the event of an aneurysmal subarachnoid hemorrhage (SAH) and vasospasm is a surgical emergency with near-universal poor outcome. Wide frontotemporal decompressive craniectomy (DECRA) is the standard life-saving surgical procedure for rapid reduction of resistant-raised intracranial tension (ICT). Seldom, it becomes difficult to control the sudden rapid rise in intracranial pressure (ICP) despite the optimal utilization of standard medical and surgical interventions. In a similar situation, authors have attempted bilateral frontotemporoparietal craniectomy (including the bony strip over the superior sagittal sinus [SSS]) with ligation of the anterior third of SSS (megacraniectomy). The patient was successfully managed with intensive care and later received cranioplasty. Authors have discussed the surgical nuances and the utility of this surgical procedure in the light of resource-stricken hospital settings.

\section{Case Report}

A 32-year-old young female presented with spontaneous SAH. She was admitted with Hunt and Hess grade II, World
Federation of Neurosurgical Societies grade 1, and Fischer's grade 3 status. Digital subtraction angiogram revealed a ruptured anterior communicating artery which is an aneurysm with the dominant left anterior cerebral artery. An aneurysm was successfully coiled. Immediate postoperative period was uneventful. On the first postcoiling day, the patient developed severe vasospasm and deteriorated to E2V2M5 status. Despite adequate medical management, the patient showed rapid neurological deterioration. Plain computed tomography (CT) head showed signs of malignant intracranial edema and global vasospasm corroborating with findings of transcranial ultrasonography. The patient received intra-arterial nimodipine with no significant improvement. External ventricular drainage was placed with continuous ICP monitoring through the right frontal horn. After a transient improvement, the patient rapidly deteriorated to decerebrate status with clinical features of Cushing's triad (ICP $>35 \mathrm{~cm}$ water). All resuscitative measures with ventilation were continued. Repeat noncontrast computed tomography (CT) head suggested diffuse brain edema, effaced gyri and sulci, compressed ventricles, and evolving ischemic changes throughout the brain ( - Fig. 1A). With due consent, the patient was taken for DECRA as a last resort to control rapid rise in ICP. 

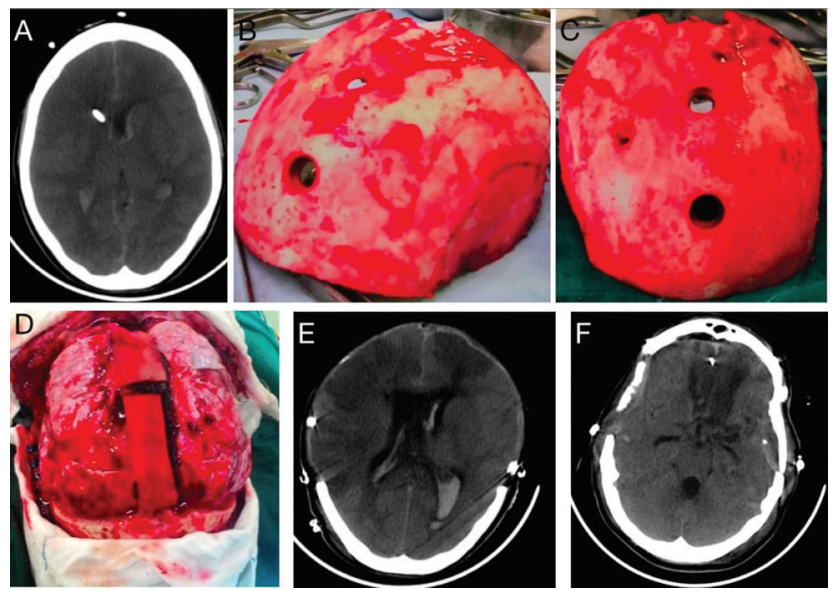

Fig. 1 (A) Preoperative noncontrast computed tomography head showing diffuse hypodensity and brain edema; $(\mathbf{B}, \mathbf{C})$ megacraniectomy bone flap showing en mass removal of bone from frontal, temporal, and parietal areas; (D) relaxed brain after durotomy and removal of bone from skull except for occipital bone; (E) postoperative noncontrast computed tomography head showing relaxed brain parenchyma; (F) postcranioplasty noncontrast computed tomography head at follow-up.

\section{Megacraniectomy}

In view of global cerebral hypodensity, the decision was taken for widest possible DECRA for rapid reduction in ICP. Options of bifrontal craniectomy and bilateral frontotemporal craniectomy were considered; however, in view of chances of the "scissoring effect on the cortical veins" at bony margins, the decision was taken for bifrontotemporoparietal craniectomy. An extended bicoronal incision placed $6 \mathrm{~cm}$ behind the coronal suture (autopsy incision) was fashioned with anterior limb just anterior to the tragus bilaterally ( - Figs. 1B, C and 2A-C).

Posteriorly, occipital and some part of parietal bone were left so that the head could be rested in the supine posture. Craniectomy margins were flush with middle cranial fossa floor bilaterally. Bone flap was gently separated from SSS. Eight burr holes were made two on either side of SSS at the posterior parietal end, two at keyholes, two posterior temporal, and last two were directly on SSS in the midline. Star-shaped durotomy was done on either side which initially released the tight brain but inadequately. The brain was bulging in the midline causing tenting pressure and kinking of the veins. Therefore, falx cerebri was cut anteriorly near crista galli after ligating SSS. As soon as falx was totally released, the brain bulge seemed to ease. The discoloring hue of the ischemic brain was released ( - Figs. 1D, E and 2D, E). Scalp flap was closed en masse.

The patient received mechanical ventilation postoperatively and gradually improved to E2VTM5 status in a week's duration. CT scan head revealed adequate decompression with infarcts in the right frontoparietal, left frontal region, and hypodensity in hypothalamic areas. With intensive care, the patient survived the violent storm of vasospasm. After a month of surgery, she received cranioplasty and was discharged to home (-Fig. 1F). At follow-up, few weeks after surgery, she was aphasic with hemiparesis of the left side.

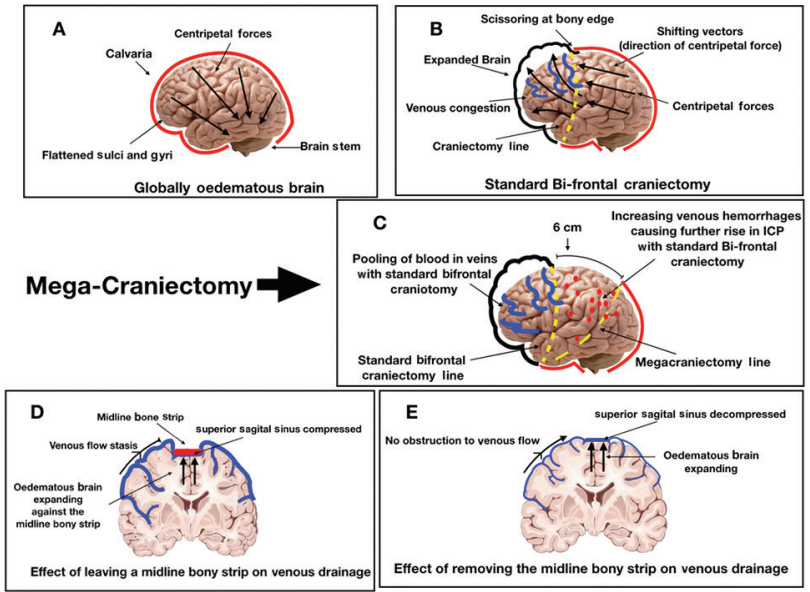

Fig. 2 (A) Centripetal forces acting on the brain leading to herniation; (B) routine bifrontal decompression causing external herniation and scissoring effect on the veins at the bone margin in cases of malignant intracranial hypertension; (C) megacraniectomy relieving malignant intracranial tension; (D) venous kinking leading to venous hypertension in preserved midline bone strip; (E) relieved venous kinking after removing midline bone from superior sagittal sinus

At 1-year follow-up, we were informed of her death at home due to a tracheostomy block because of a mucus plug.

\section{Discussion}

Refractory or malignant IHT is generally seen in cases of diffuse traumatic brain injury (TBI) or aneurysmal SAH, or malignant infarcts that frequently lead to devastating and irreversible consequences, even fatality. Standard protocol-based management of malignant ICT demands stepwise management. However, in settings of rapid neurological deterioration, unprecedented decisions might be warranted to prevent irreversible and lethal consequences. In case of TBI or unilateral infarct or bleed, it is easy to decide the site of decompression but in case of diffuse brain edema and obliteration of basal cisterns, it is quite difficult to decide the approach and side of decompression. Some studies have advocated bifrontal craniotomy, especially in the pediatric age group. ${ }^{1}$ In spite of best possible efforts, the outcome of patients with refractory IHT is quite poor with almost $34 \%$ mortality, $40 \%$ severe disability including vegetative status, $13 \%$ moderate disability, and only $3 \%$ good outcome in the patients. ${ }^{2,3}$

Doing a wide DECRA as a treatment for IHT is well-recognized since the dawn of neurosurgery. The procedures include uni- or bilateral frontal decompression or temporal decompression, or circumferential hemicraniectomy. ${ }^{4-6}$ The American Association of Neurological Surgeons has recommended DECRA for patients with TBI and refractory ICT if some or all of the following criteria were met:

1. Diffuse cerebral swelling on cranial CT imaging.

2. Within 48 hours of injury. 
3. No episodes of sustained, raised ICP $>40 \mathrm{~mm} \mathrm{Hg}$ before surgery.

4. Glasgow coma score (GCS) $>3$ at some point subsequent to injury.

5. Secondary clinical deterioration.

6. Evolving cerebral herniation syndrome. ${ }^{7}$

We did a megacraniectomy in our patient, which included doing bifrontal and bitemporal decompression including the part of the parietal bone on both sides by lifting a single large flap of bone; additionally, we ligated SSS and fractured crista galli. We did not attempt basal cisternostomy because of significant brain edema. We could not find any study mentioning division of SSS but that decision was the need of that moment because of severe intraoperative brain bulge not getting controlled in spite of best efforts. We did not find any similar study describing identical technique; hence, the comparison with standard techniques cannot be done just on the basis of an anecdotal case. Our patient did not become conscious but improved from E1VTM2 status to E2VTM5 status and survived more than a year which may not have been otherwise possible.

Large decompression provides immediate and effective control of ICP than other types of decompressive surgeries, as it provides adequate space for both hemispheres to expand, which relieves subfalcine and transtentorial brain herniation, and early curtailment of medical measures for lowering ICP. ${ }^{8-10}$ The timing of intervening surgically is considered as an important predictor of outcome; ideally, the procedure should be performed before the evolution of brain infarction or development of secondary brain damage. Of the previous factors, the amount of primary brain injury, timing of surgery, level of ICP, and GCS score before surgery are considered as predictive of outcome after large DECRA. ${ }^{11,12}$

In the original technique described by Polin et $\mathrm{al}^{13}$ which was also used in the DECRA ${ }^{14}$ trial, the surgery comprised of decompressive craniectomies with a singlefrontotemporal bone flap extending across the midline. The temporalis muscle was reflected inferiorly. Burr holes were located at either side of the sagittal sinus at the posterior extent and bilaterally at the keyhole and at the root of the zygoma. This created a large bifrontal craniectomy defect extending posteriorly onto the parietal bones 3 to 5 $\mathrm{cm}$ posterior to the coronal sutures. However, there were significant issues in the technique, as they did not divide the sagittal sinus and falx cerebri, limiting the procedural efficacy for lowering intracranial pressure. Hence, the conclusions of the trial that better functional outcomes were obtained when standard care was provided (including inducement of coma with a barbiturate in $77 \%$ of patients receiving standard care) than through craniectomy are not generalizable, as can be seen by the number of issues raised against the trial results. ${ }^{15}$ We firmly believe that in the case of desperate situations, a megacraniectomy of this nature may help in saving lives.

\section{Conclusion}

Whether such an extensive procedure was needed or not, but at that particular moment, it was used as the last rescue resort. In this race against time and odds, we were forced to go for the largest possible decompression. The three key determining steps in malignant brain edema were the separation of bone from SSS, flush bitemporal decompression, and cutting of the anterior third of falx. All of these steps were done with the utmost care. We do not have enough data to support our approach to be used on a regular basis, but it is a new approach, which should be investigated further with adequate follow-up to support or refute. Once you appreciate the brain getting released from the tightly holding dura and falx, it is a great sigh of relief that the job is appropriately done.

\section{Conflict of Interest}

None declared.

\section{References}

1 Honeybul S, Ho KM, Lind CR, Gillett GR. Decompressive craniectomy for diffuse cerebral swelling after trauma: long-term outcome and ethical considerations. J Trauma 2011;71(1):128-132

2 Marshall LF, Marshall SB, Klauber MR, et al. A new classification of head injuries based on computerized tomography. J Neurosurg 1991;75(Suppl Nov.1991):S1-S66

3 Marshall LF, Becker DP, Bowers SA, et al. The national traumatic coma data bank. Part 1: design, purpose, goals, and results. J Neurosurg 1983;59(2):276-284

4 Marshall LF. Head injury: recent past, present, and future. Neurosurgery 2000;47(3):546-561

5 Alexander E, Ball MR, Laster DW. Subtemporal decompression: radiological observations and current surgical experience. $\mathrm{Br}$. J Neurosurg 1987;1(4):427-433

6 Miyazaki Y, Hirai H, Hachisu Y, Takada I. [Bifrontal external decompression for traumatic brain edema]. Shujutsu 1966;20(10):845-852

7 Guidelines for the management of severe head injury. Brain trauma foundation, American Association of Neurological Surgeons, Joint Section on Neurotrauma and Critical Care. J Neurotrauma 1996;13(11):641-734

8 Ruf B, Heckmann M, Schroth I, et al. Early decompressive craniectomy and duraplasty for refractory intracranial hypertension in children: results of a pilot study. Crit Care 2003;7(6):R133-R138

9 Elwatidy S, El-dawlatly A, Jamjoom ZA, Elgamal E. Use of transcranial cerebral oximeter as indicator for bifrontal decompressive craniotomy. Internet J Anaesthiol 2004;8(2):1-3

10 Taylor A, Butt W, Rosenfeld J, et al. A randomized trial of very early decompressive craniectomy in children with traumatic brain injury and sustained intracranial hypertension. Childs Nerv Syst 2001;17(3):154-162

11 Kjellberg RN, Prieto A, Jr. Bifrontal decompressive craniotomy for massive cerebral edema. J Neurosurg 1971;34(4):488-493

12 Ziai WC, Port JD, Cowan JA, Garonzik IM, Bhardwaj A, Rigamonti D. Decompressive craniectomy for intractable cerebral edema: experience of a single center. J Neurosurg Anesthesiol 2003;15(1):25-32 
13 Polin RS, Shaffrey ME, Bogaev CA, et al. Decompressive bifrontal craniectomy in the treatment of severe refractory posttraumatic cerebral edema. Neurosurgery 1997;41(1):84-92,discussion92-94

14 Cooper DJ, Rosenfeld JV, Murray L, et al. DECRA Trial Investigators; Australian and New Zealand Intensive Care Society
Clinical Trials Group. Decompressive craniectomy in diffuse traumatic brain injury. N Engl J Med 2011;364(16):1493-1502

15 Hutchinson PJ, Timofeev I, Kolias AG, et al. Decompressive craniectomy for traumatic brain injury: the jury is still out. Br. J Neurosurg 2011;25(3):441-442 